Research Article

\title{
Time-Fractional Model of Chloride Diffusion in Concrete: Analysis Using Meshless Method
}

\author{
Ling Yao $\mathbb{D}$, Lixia Ren, and Guoli Gong \\ Department of Resources and Environment, Shanxi Institute of Energy, No. 63, University Street, University Park, \\ Shanxi Province, China \\ Correspondence should be addressed to Ling Yao; yaoling318@163.com
}

Received 22 March 2020; Revised 2 May 2020; Accepted 19 May 2020; Published 3 June 2020

Academic Editor: Georgios I. Giannopoulos

Copyright $\odot 2020$ Ling Yao et al. This is an open access article distributed under the Creative Commons Attribution License, which permits unrestricted use, distribution, and reproduction in any medium, provided the original work is properly cited.

Chloride diffusion is the major factor that affects the life of concrete structures. The time-fractional order equation can be used to describe anomalous diffusion in reinforced concrete. In this work, a time-fractional model of chloride diffusion is solved via the meshless method. The Element-Free Galerkin (EFG) meshless method does not require meshing. One-dimensional and two-dimensional numerical examples are presented. Numerical results are in good agreement with theoretical solutions. The initiation time of corrosion is predicted in the presented model. Simulation results are compared with experimental data. The good agreement between EFG and experimental data indicates that time-fractional chloride diffusion in concrete can be modeled effectively by using the EFG method. This method is beneficial for further research on anomalous chloride diffusion in concrete.

\section{Introduction}

Reinforced concrete (RC) structures in coastal areas may suffer from rebar corrosion, and chloride ingression is the main factor that affects the durability of concrete structures [1-4]. Chloride diffusion can be described by traditional models via the diffusion equation that conforms to Fick's law under the assumption that the concrete is a homogeneous and porous material [5]. However, the concrete material is actually not uniform and stable; some observers have found that the actual chloride diffusion process does not satisfy the traditional diffusion model and the chloride diffusion process particularly exhibits time-dependent feature in engineering practice, which is called anomalous diffusion $[6,7]$. The chloride diffusion mechanism in concrete is highly complicated because of wicking, chloride binding, permeation, and absorption, Furthermore, this case is an anomalous diffusion process, in which particles diffuse slower than the normal diffusion and its time-dependent features cannot be accurately reflected by traditional models [8].
Anomalous diffusion has sparked wide interest in the fields of physics and engineering in recent years [9]. Anomalous diffusion process is essentially a process with memory in time and nonlocality in space, the definition of the integral derivative limit is localized. Moreover, traditional diffusion equations can no longer accurately describe anomalous diffusion behaviors. Fractional-order equations are mathematical models that have been proved to accurately reflect this type of anomalous diffusion phenomenon.

Fractional differential equations are a generalization of integer-order differential equations, and their application prospect is broad. Fractional complete equations have been used in viscoelasticity, dynamics, leaky pools, cybernetics, and statistics in recent decades $[10,11]$. However, they are not widely applied in modeling chloride diffusion in concrete. The time-fractional diffusion model can be applied to predict chloride diffusion. It is solved through the finite difference method. At present, the finite element method (FEM) and the finite difference method (FDE) are commonly used for the numerical solution of fractional diffusion equations $[12,13]$. FEM or FDE must be divided into grids 
during solution. Grid meshing will require considerable calculation time when a structure is highly complicated.

Numerous meshless methods that avoid meshing have been developed. In recent years, these methods have been widely used in different areas, such as elasticity, thermal conduction, and fracture mechanics. The meshless method has been used to model chloride diffusion in concrete only by [14-17]. Among these meshless methods, the ElementFree Galerkin (EFG) meshless method has been used successfully to solve the problems of plates and shells $[18,19]$, fracture mechanics and wave propagation [20-23], and electromagnetic field analysis [24]. However, this method has not been used for the time-fractional model analysis of concrete.

In the present work, the EFG meshless method is used to solve the time-fractional model of chloride diffusion in concrete. The EFG meshless method does not require element connectivity, and integration over the solution domain requires only the simple integration of cells to obtain the solution. The governing differential equation is obtained using the Galerkin weak form, and the moving least-square (MLS) approximation is adopted in EFG [25]. The essential boundary conditions to be imposed are directly approached as a penalty in this work.

The structure of this paper is as follows: in Section 2, the MLS approximation is discussed briefly. The discrete timefractional chloride diffusion model is presented in EFG form in Section 3, one-dimensional (1D) and two-dimensional (2D) numerical examples are then solved, discussed, and analyzed, and EFG and experimental results are compared in Section 4. The conclusion is presented in the following section.

\section{MLS Approximants}

The unknown concentration function $C(x)$ is approximated by the MLS approximant $C^{h}(x)$. It can be written as follows [26]:

$$
C^{h}(x)=\sum_{k=1}^{m} p_{k}(x) a_{k}(x)=P^{\mathrm{T}}(x) a(x),
$$

where $m$ is the number of terms in the basis, $p_{k}(m)$ is the monomial base function, and $a_{k}(x)$ is an unknown coefficient.

The coefficient is obtained by minimizing function $J$ and is written as follows:

$$
\begin{aligned}
J & =\sum_{i=1}^{N} w_{i}\left(x-x_{i}\right)\left[C^{h}\left(x, x_{i}\right)-C\left(x_{i}\right)\right] \\
& =\sum_{i=1}^{N} w_{i}\left(x-x_{i}\right)\left[P^{\mathrm{T}}\left(x_{i}\right) \mathrm{a}(x)-C\left(x_{i}\right)\right]^{2},
\end{aligned}
$$

where $w_{i}\left(x-x_{i}\right)$ is a weight function. It is important in the EFG method. The weight function $w_{i}\left(x-x_{i}\right)$ is nonzero over a small neighborhood of $x_{i}$, which is called the domain of influence of node.

$$
\begin{aligned}
& \text { Let } \frac{\partial J}{\partial \mathrm{a}}=A(x) \mathrm{a}(x)-B(x) C=0, \\
& \text { Such that } A(x)=\sum_{i=1}^{n} w_{i}\left(x-x_{i}\right) P\left(x_{i}\right) P^{\mathrm{T}}\left(x_{i}\right) \text {, } \\
& B(x)=\left[w_{1}\left(x-x_{1}\right) P\left(x_{1}\right), w_{2}\left(x-x_{2}\right) P\left(x_{2}\right) \ldots\right. \\
& \left.w_{n}\left(x-x_{n}\right) P\left(x_{n}\right)\right], \\
& \mathrm{a}(x)=A^{-1}(x) B(x) C .
\end{aligned}
$$

In accordance with equations (1) and (4), we have

$$
C(x) \approx C^{h}(x)=P^{\mathrm{T}}(x) A^{-1}(x) B(x) .
$$

\section{Discretization Scheme}

3.1. Time-Fractional Chloride Diffusion Model in Concrete. Chloride diffusion in concrete is assumed to follow Fick's second law. The equation is written as

$$
\frac{\partial C}{\partial t}=D_{x} \frac{\partial^{2} C}{\partial x^{2}}+D_{y} \frac{\partial^{2} C}{\partial y^{2}},
$$

where $C=C(x, y, t)$ represents concentration; $t$ is time; and $D_{x}$ and $D_{y}$ are diffusion coefficients in the $x$ and $y$ directions, respectively.

When $D$ is a constant in one dimension, the analytical solution of equation (6) is expressed as

$$
C=C_{s}\left[1-\operatorname{erf}\left(\frac{x}{2 \sqrt{D t}}\right)\right],
$$

where $C_{s}$ is the chloride concentration at the exposure surface and erf (.) is the error function.

We fully use the time-fractional diffusion equation to establish the time-fractional model of chloride ion diffusion in concrete as follows:

$$
\begin{array}{r}
\frac{\partial^{\alpha} C}{\partial t^{\alpha}}=D_{\alpha x} \frac{\partial^{2} C}{\partial x^{2}}+D_{\alpha y} \frac{\partial^{2} C}{\partial y^{2}}, \\
C(x, y, 0)=C_{0} \quad \text { in } \Omega, \\
C=C_{s} \quad \text { on } \Omega_{1},
\end{array}
$$

where $D_{\alpha x}$ and $D_{\alpha y}$ are the diffusion coefficients in the timefractional model of chloride ion diffusion in concrete.

In equation (8), $\partial^{\alpha} C / \partial t^{\alpha}$ is the Caputo fractional derivative of the order $\alpha \quad(0<\alpha \leq 1)$, which is defined as follows:

$$
\frac{\partial^{\alpha} C}{\partial t^{\alpha}}=\frac{1}{\Gamma(1-\alpha)} \int_{0}^{t} \frac{\partial C}{\partial \varsigma} \frac{\mathrm{d} \varsigma}{(t-\varsigma)^{\alpha}},
$$

where $\Gamma(\cdot)$ is the gamma function. Equation (8) is equivalent to equation (6), when $\alpha=1$. 
3.2. Time-Fractional Derivative Approximation. Defining $\Delta t$ as the time interval and $t_{k}=n \Delta t,(n=1,2,3 \ldots)$, the timefractional derivative at $t=t_{n+1}$ can be approximated as

$$
\begin{aligned}
& \frac{\partial^{\alpha} C\left(\mathbf{x}, t_{n+1}\right)}{\partial t^{\alpha}}=\frac{1}{\Gamma(1-\alpha)} \int_{0}^{t_{n+1}} \frac{\partial C(\mathbf{x}, \varsigma)}{\partial t} \frac{\mathrm{d} \varsigma}{\left(t_{n+1}-\varsigma\right)^{\alpha}} \\
& =\frac{1}{\Gamma(1-\alpha)} \sum_{j=0}^{n}\left(\frac{C\left(\mathbf{x}, t_{j+1}\right)-C\left(\mathbf{x}, t_{j}\right)}{\Delta t}+R_{1}\right) \int_{t_{j}}^{t_{j+1}} \frac{\mathrm{d} \varsigma}{\left(t_{n+1}-\varsigma\right)^{\alpha}} \\
& =\frac{1}{\Gamma(2-\alpha)} \sum_{j=0}^{n} \frac{C\left(\mathbf{x}, t_{n-j+1}\right)-C\left(\mathbf{x}, t_{n-j}\right)}{\Delta t^{\alpha}}\left[(j+1)^{1-\alpha}-j^{1-\alpha}\right]+R_{2} \\
& =A(\alpha)\left[C\left(\mathbf{x}, t_{n+1}\right)-\left(\mathbf{x}, t_{n}\right)\right] \\
& \quad+A(\alpha) \sum_{j=1}^{n} B(j)\left[C\left(\mathbf{x}, t_{n-j+1}\right)-\left(\mathbf{x}, t_{n-j}\right)\right]+R_{2},
\end{aligned}
$$

where $R_{1}$ and $R_{2}$ are the truncation errors and $\Gamma(\cdot)$ is the gamma function. And,

$$
\begin{aligned}
& A(\alpha)=\Delta t^{-\alpha} \frac{1}{\Gamma(2-\alpha)}, \\
& B(j)=(j+1)^{1-\alpha}-j^{1-\alpha} .
\end{aligned}
$$

3.3. Space Discretization in EFG Meshless Method. The weak form of equation (6) is obtained via variation as follows:

$$
\delta \Pi(C)=\int \delta C \frac{\partial^{\alpha} C}{\partial t^{\alpha}}-D_{x} \frac{\partial(\delta C)}{\partial x} \frac{\partial C}{\partial x}-D_{y} \frac{\partial(\delta C)}{\partial y} \frac{\partial C}{\partial y} \text {. }
$$

By using the penalty function method to address the boundary conditions in this work, equation (14) is obtained as

$$
\delta \Pi^{*}(C)=\delta \Pi(C)+\left.\operatorname{alfa}\left(C-C_{s}\right)\right|_{\Omega_{1}},
$$

where alfa is the penalty parameter and equals $10^{3}-10^{8}$.

In accordance with equation (5), we can obtain the following expressions:

$$
C(x) \approx C^{h}(x)=\sum_{i=1}^{N} \Phi_{i} C_{i}=\Phi C
$$

where $\Phi$ is the shape function and is written as follows:

$$
\begin{aligned}
\Phi(x)= & {\left[\Phi_{1}(x), \Phi_{2}(x), \Phi_{3}(x), \ldots, \Phi_{n}(x)\right]=P^{T} A^{-1}(x) B(x), } \\
\frac{\partial C^{h}}{\partial x}= & \sum_{i=1}^{N} \frac{\partial \Phi_{i}(x)}{\partial x} C_{i}=\Phi_{x}(x) C, \\
\Phi_{x}(x)= & \frac{\partial \Phi(x)}{\partial x}=\left[\frac{\partial \Phi_{1}(x)}{\partial x}, \frac{\partial \Phi_{2}(x)}{\partial x}, \frac{\partial \Phi_{3}(x)}{\partial x}, \cdots, \frac{\partial \Phi_{N}(x)}{\partial x}\right] \\
& =\left[\Phi_{1, x}(x), \Phi_{2, x}(x), \Phi_{3, x}(x), \cdots, \Phi_{N, x}(x)\right], \\
\frac{\partial^{\alpha} C^{h}}{\partial t^{\alpha}}= & \Phi(x)\left[A(\alpha)\left(C_{n+1}-C_{n}\right)+A(\alpha) \sum_{j=1}^{n} B(j)\left(C_{n-j+1}-C_{n-j}\right)\right] .
\end{aligned}
$$

Substituting the above three equations into equation (14), we obtain

$$
\delta C^{\mathbf{T}} M\left[A(\alpha)\left(C_{n+1}-C_{n}\right)+U\right]+\delta C^{\mathbf{T}} K C+\delta C^{\mathbf{T}} H C-\delta C^{\mathbf{T}} Q=0,
$$

where

$$
\begin{aligned}
M_{i j} & =\int_{\Omega} \Phi_{i}(x) \Phi_{j}(x) \mathrm{d} \Omega \\
U & =A(\alpha) \sum_{j=1}^{n} B(j)\left(C_{n-j+1}-C_{n-j}\right) \\
K & =\int_{\Omega}\left[D_{\alpha x} \Phi_{i, x}(x) \Phi_{j, x}(x)+D_{\alpha y} \Phi_{i, y}(y) \Phi_{j, y}(y) \mathrm{d} \Omega\right] \\
H & =\operatorname{alfa} \int_{\Omega_{1}} \Phi_{i}(x) \Phi_{j}(x) \mathrm{d} \Omega_{1} .
\end{aligned}
$$

In accordance with the arbitrariness of $\delta C^{\mathbf{T}}$, via the time central difference method, then

$$
M\left[A(\alpha)\left(C_{n+1}-C_{n}\right)+U\right]+(K+H) \frac{C_{n+1}+C_{n}}{2}-\frac{Q_{n+1}+Q_{n}}{2}=0 .
$$

Simplifying equation (19) yields equation (20) that is the discrete expression of the time-fractional model:

$$
M_{\text {eff }} C_{n+1}=K_{\text {eff }} C_{n}+Q_{\text {eff }},
$$

where

$$
\begin{gathered}
M_{\mathrm{eff}}=M A(\alpha)+\frac{K+H}{2}, \\
\mathrm{~K}_{\mathrm{eff}}=\mathrm{MA}(\alpha)-\frac{\mathrm{K}+\mathrm{H}}{2}, \\
Q_{\mathrm{eff}}=\frac{Q_{n+1}+Q_{n}}{2}-M U .
\end{gathered}
$$

\section{Numerical Examples}

The application of the time-fractional model of the EFG meshless method is illustrated using $1 \mathrm{D}$ and $2 \mathrm{D}$ examples of chloride diffusion in concrete. MATLAB codes are developed to obtain the EFG meshless method.

4.1. 1D Chloride Diffusion in Concrete. The example is a $0.15 \mathrm{~m} \times 0.15 \mathrm{~m}$ concrete block. The left boundary is $5 \%$ (chloride concentration). In the first example, the diffusion coefficient is assumed to be constant $D_{\alpha}=1.5768 \times 10^{-12} \mathrm{~m}^{2} / \mathrm{s}$, and in the second example, $D$ is a time-dependent function $D=D_{0}\left(t_{0}\right)^{m}$, where $D_{0}$ is the diffusion coefficient at reference time, $t_{0}$ is reference time, and $m$ is an age factor. The initial chloride concentration is zero. When $\alpha=1$, the time-fractional chloride (TFC) diffusion model in concrete is the same as the traditional chloride (TC) diffusion model. For the purpose of convergence studies, the root mean square (RMS) error is 
defined as $R=(1 / N) \sqrt{\sum_{i=1}^{N} C\left(x_{i}\right)-C^{E}\left(x_{i}\right)}$, where $N$ is the number of nodes, $C\left(x_{i}\right)$ is the calculation with meshless methods, and $C^{E}\left(x_{i}\right)$ denotes the analytical solution.

Example 1. The block is divided into 31 nodes. When $\alpha=1$, the EFG meshless method in the TFC model, based on the weighted least-squares (MWLS) method [17] and FEM method in the TC model, is adopted. C20e and C50e are analytical solutions according to equation (7), C20 $\mathrm{m}$ and C50 m denote chloride concentration in the TFC model, C20 mw and C50 mw mean chloride concentration from MWLS at exposure times of 20 and 50 years, and C20f and C50f denote chloride concentration from FEM in Figure 1. The results of the TFC model and TC model are consistent with the analytical values presented in Figure 1 . The RMS error is $0.9543 \%$ in TFC, RMS error of MWLS is $1.2984 \%$, and RMS error of FEM is $0.9869 \%$ in the TC model when $t=20$ years. The errors between the simulated results and the analytical results are very small. This result shows that the simulation of the TFC model using the meshless method is correct.

Figure 2 shows the change in chloride concentration at different depths with the time-fractional order $\alpha$. Chloride concentration gradually decreases with the increment in depth, as shown in Figure 2. The chloride concentration at the depth of $5 \mathrm{~cm}$ is $0.4502 \%, 1.0196 \%$, and $1.901 \%$ when the value of $\alpha$ is $0.5,0.7$, and 0.9 , respectively. The chlorine concentration at the same depth when $t=50$ years increases with the increase in $\alpha$. Large values of $\alpha$ indicate strong chlorine permeability and correspond to high chloride concentration.

Critical chloride ion content $\left(C_{t}\right)$ is selected as $0.5 \%$, and a rebar is assumed to exist at a depth of $5 \mathrm{~cm}$. The initiation period of corrosion for the start of the rebar is shown in Table 1. The predictions for the initiation periods of corrosion are clearly different. These results show that chloride concentration increases with the increase in $\alpha$. The results are almost the same when $\alpha=1.0$ in TFC and the analytical solution in TC.

Example 2. The coefficient efficient is a time-function $D=D_{0}\left(t_{0}\right)^{m}$. In this example, $D_{0}=1.5768 \times 10^{-12} \mathrm{~m}^{2} / \mathrm{s}, t_{0}=28$ days and $m=0.1$. It means $D_{\alpha}=D_{0}\left(t_{0}\right)^{m} \Gamma(\alpha)$ and $\alpha=1-m$ in the TFC model according to reference [8]. The parameters $D_{\alpha}$ and $\alpha$ can be obtained by mean square displacement (MSD) and some data fitting techniques, such as least-square fitting of experimental data [27]. The values of the remaining parameters are the same as in Example 1.

As shown in Figure 3, the change trend of chlorine concentration is decreasing with depth increasing. The chloride concentration of the same depth is increasing with the increase of the $\alpha$ value when $t=20$ years. The greater the value of $\alpha$, the greater the concentration of chloride.

4.2. $2 D$ Chloride Diffusion in Concrete. Figure 4 depicts a $0.15 \mathrm{~m} \times 0.15 \mathrm{~m}$ concrete slab. The left and bottom boundaries are $0.6 \%$, the remaining edges are zero, and the chloride efficient $D=9.38 \times 10^{-12} \mathrm{~m}^{2} / \mathrm{s}$.

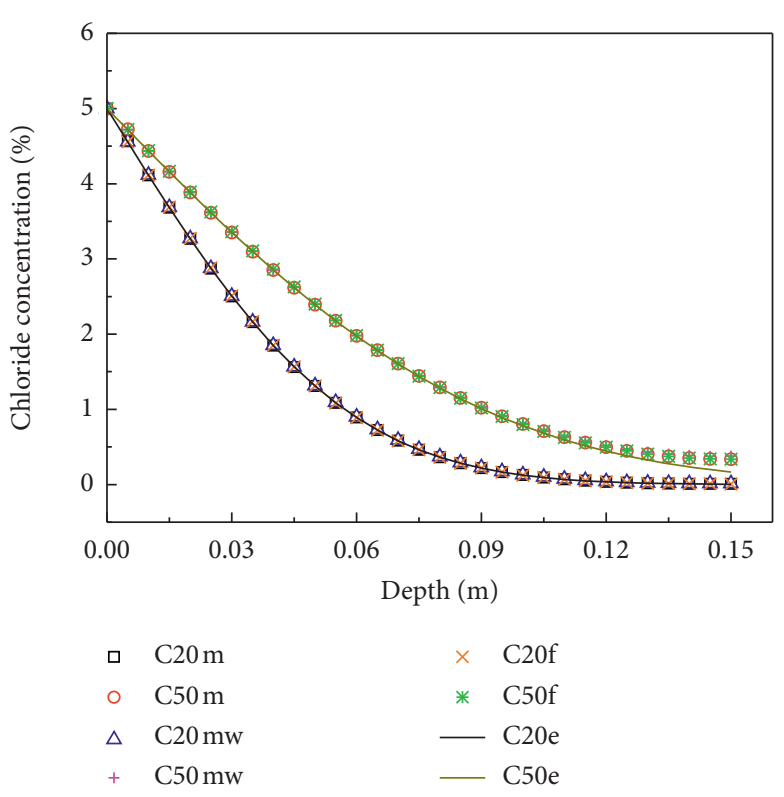

FIGURE 1: Change in chloride concentration with depth.

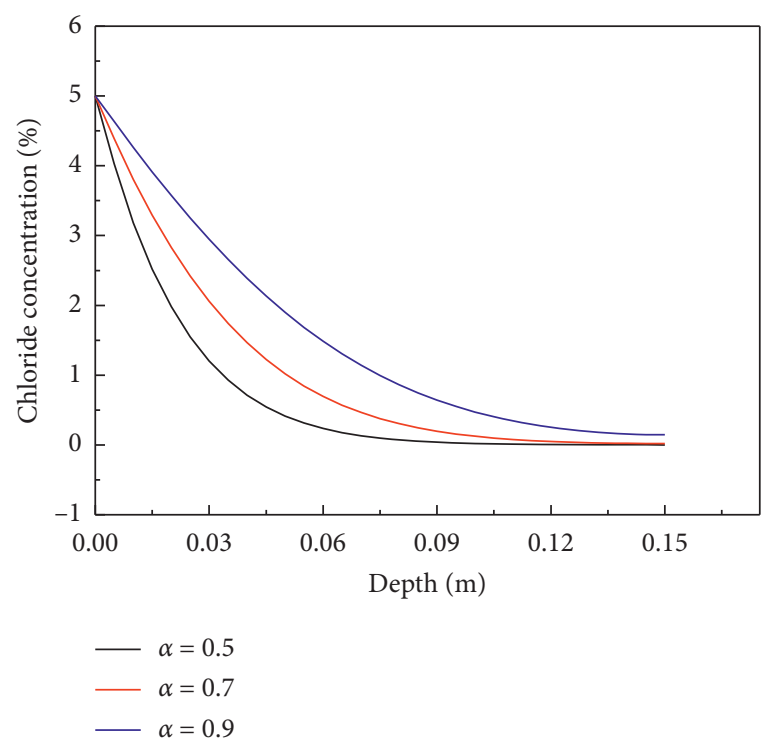

Figure 2: Chloride concentration-depth with different $\alpha$ in TFC $(t=50$ years $)$.

TABLE 1: Initiation period of corrosion under different $\alpha$.

\begin{tabular}{lcccccc}
\hline$\alpha$ & 0.2 & 0.4 & 0.6 & 0.8 & 1.0 & Exact \\
\hline Initiation period (year) & 18.30 & 16.30 & 7.40 & 5.40 & 4.60 & 4.67 \\
\hline
\end{tabular}

Example 3. The regular distribution of $31 \times 31$ nodes is selected in the $\Omega$ domain, and when the diffusion coefficient $D$ is a constant, the analytical solution is as follows [28]:

$$
C=C_{s}\left[1-\operatorname{erf}\left(\frac{x}{2 \sqrt{D_{x} t}}\right) \operatorname{erf}\left(\frac{y}{2 \sqrt{D_{y} t}}\right)\right] \text {. }
$$




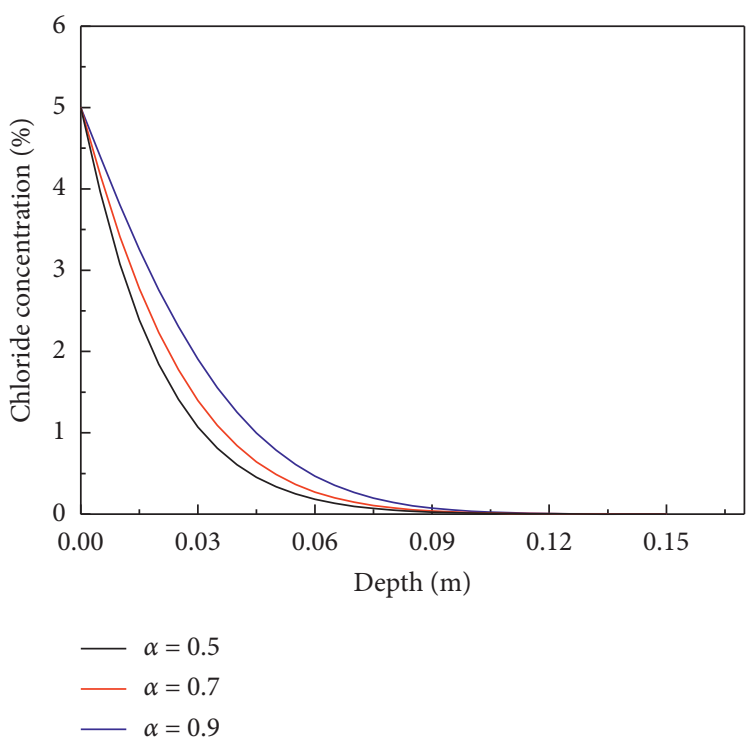

FIgURE 3: The change of chloride concentration with different $\alpha$ ( $t=20$ years).

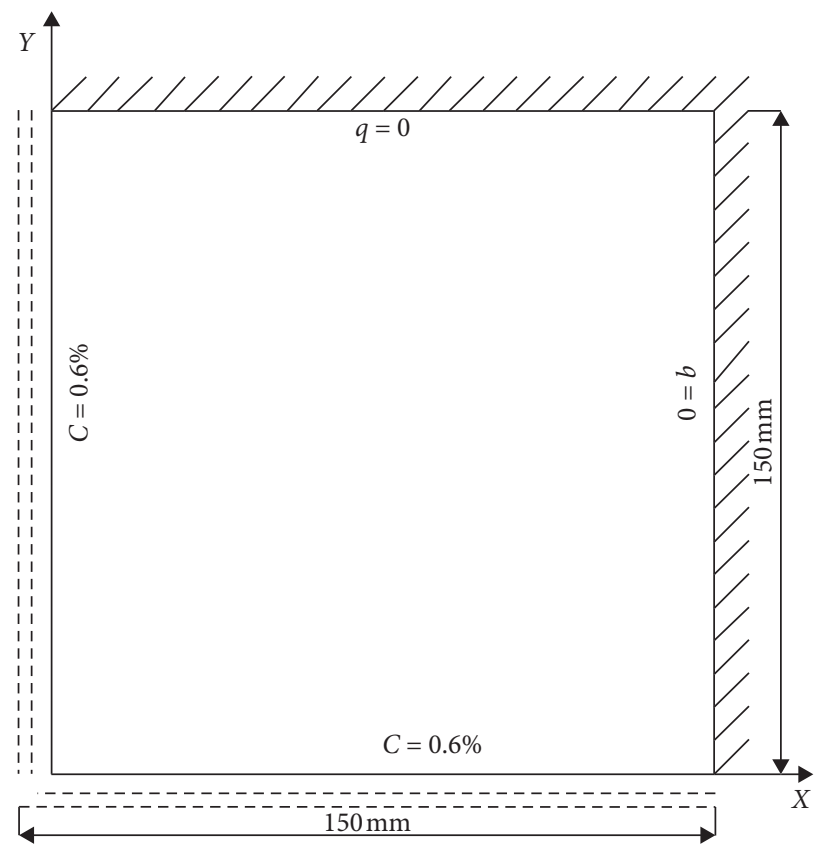

Figure 4: 2D concrete slab.

The chloride concentration at the depth of $75 \mathrm{~mm}$ when $\alpha=1$ in the TFC model and $t$ is 10 years is shown in Table 2 . As shown in Table 2, the error between the simulated value and the theoretical value is small when the depth is $75 \mathrm{~mm}$. This result confirms the correctness of the simulation method.

Figure 5 shows the distribution of chloride concentration when $\alpha$ is a different value in the TFC model and $t$ is 20 years. As Figure 5 shows, in line with the actual situation, the numerical results have the same diffusion tendency. The chloride concentration value at the same position increases as $\alpha$ increases.
The initiation period for the start of a rebar when $C_{t}=$ $0.1 \%$ at a depth of $75 \mathrm{~mm}$ is shown in Table 3 . The initiation period is the same as the theoretical value when $\alpha=1.0$. However, the initiation period is different when $a$ is different. Similar to the initiation period for the 1D example, the initiation period is large when $\alpha$ is small.

Example 4. The coefficient efficient is a time-function $D=D_{0}\left(t_{0}\right)^{m}$, the left and bottom boundaries are $3 \%$, and other conditions are the same as in Example 3. $D_{0}=$ $9.38 \times 10^{-12} \mathrm{~m}^{2} / \mathrm{s}, t_{0}=28$ days and $m=0.2$. It means $D_{\alpha}=$ $D_{0}\left(t_{0}\right)^{m} \Gamma(\alpha)$ and $\alpha=0.8$ in the TFC model. Figure 6 is the 
TABLE 2: Chloride concentration of $75 \mathrm{~mm}$ depth ( $t=10$ years).

\begin{tabular}{cccc}
\hline$x$ location $(\mathrm{mm})$ & $y$ location $(\mathrm{mm})$ & Simulated value $(\%)$ & Analytical value $(\%)$ \\
\hline & 30 & 0.4802 & 0.4779 \\
75 & 60 & 0.3711 & 0.3728 \\
& 90 & 0.3017 & 0.2950 \\
& 120 & 0.2572 & 0.2455 \\
\hline
\end{tabular}

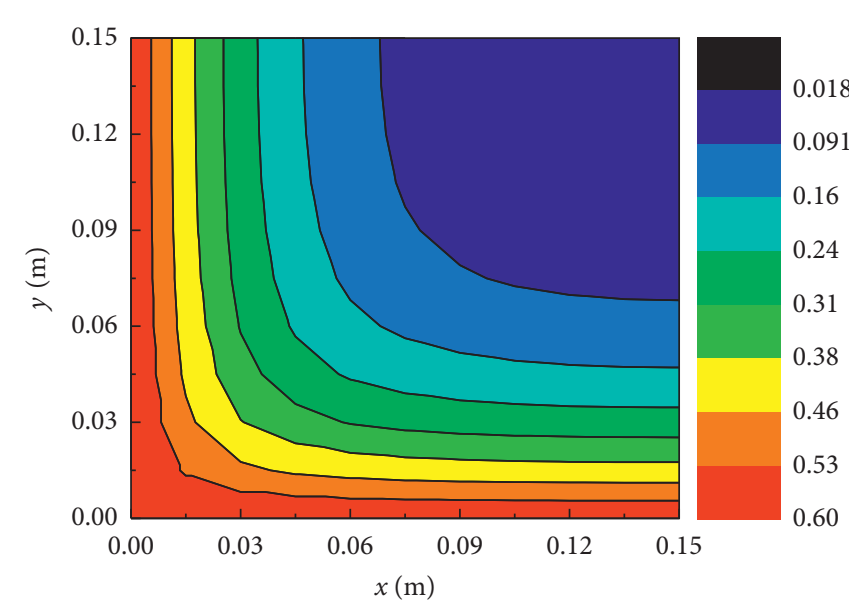

(a)

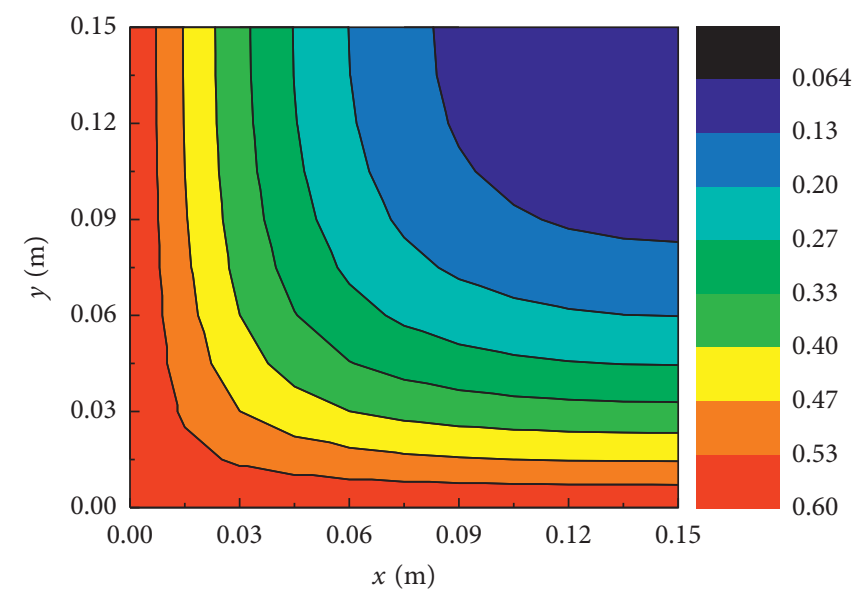

(b)

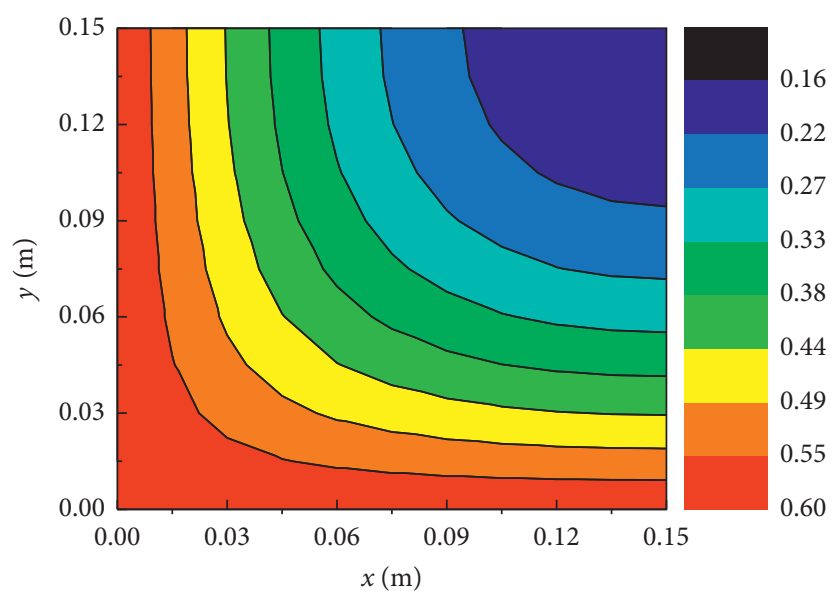

(c)

Figure 5: Distribution of chloride concentration ( $t=20$ years). (a) $\alpha=0.4$, (b) $\alpha=0.6$, and (c) $\alpha=0.8$.

TABLE 3: Initiation period of corrosion under different $\alpha$.

\begin{tabular}{lccccc}
\hline$\alpha$ & 0.4 & 0.6 & 0.8 & 1.0 & Exact \\
\hline Initiation period (year) & 14.10 & 5.75 & 3.85 & 3.15 & 3.25 \\
\hline
\end{tabular}

change of chloride concentration at the diagonal of the concrete slab. The chloride concentration of $60 \mathrm{~mm}$ is $0.7353 \%$ and $1.2464 \%$ after 5 and 10 years in the TFC model, respectively. The chloride concentration increases with time.
4.3. Experimental Numerical Simulation Using TFC Model. To further verify the correctness of the proposed model, we conducted a comparison between the simulated values and the experimental values in references [29]. The simulated 


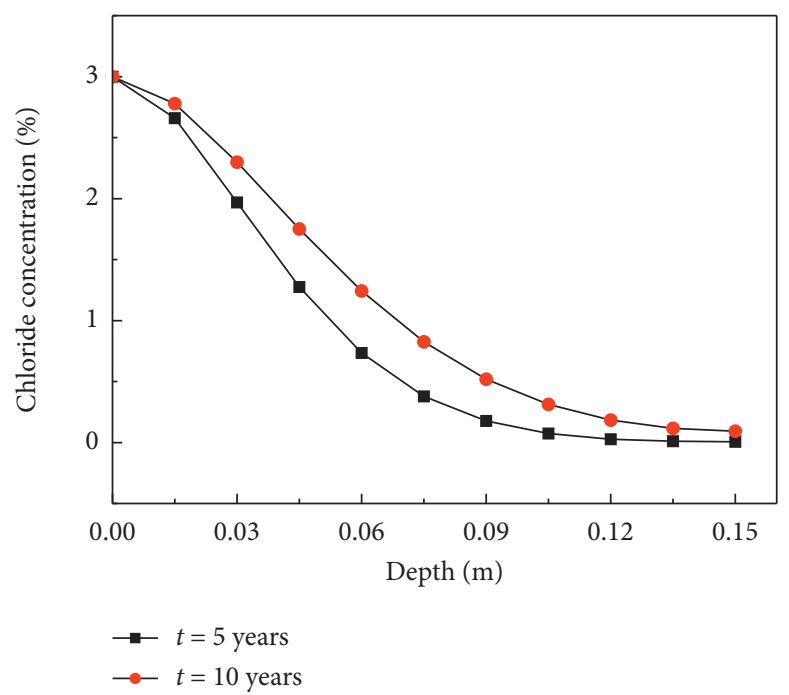

FIgURE 6: The chloride concentration at the diagonal of the concrete slab.

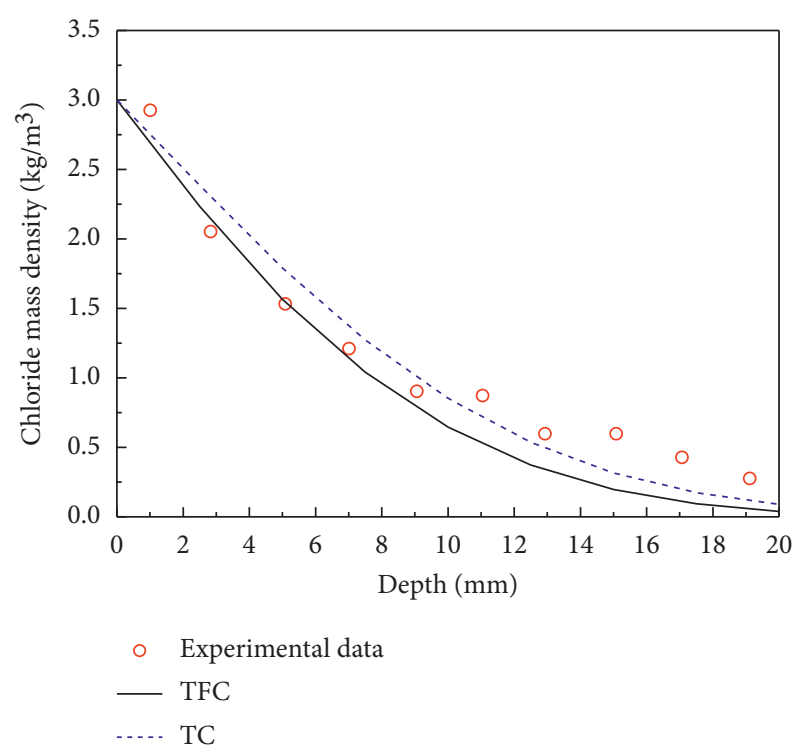

(a)

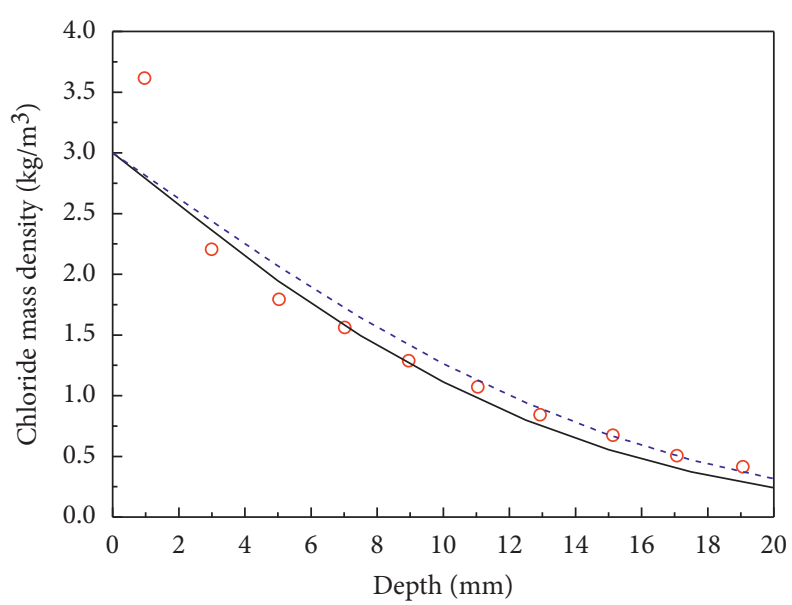

- Experimental data

- TFC

TC

Figure 7: Experimental and simulated distribution of mass densities of chloride. (a) $t=90$ days and (b) $t=180$ days.

results come from the TFC model in the EFG method and the TC model calculated by the MWLS method, respectively. The tests on four types of concrete mix were described in [29]. We choose the C3 (cement CEM I $42.5 \mathrm{~N} / \mathrm{SR} 3 / \mathrm{NA}$ ) sample, surface chloride mass density is $3 \mathrm{~kg} / \mathrm{m}^{3}$, and the chloride diffusion coefficient $D=5.2 \times 10^{-12} \mathrm{~m}^{2} / \mathrm{s}$ at $t=90$ days and $D=4.73 \times 10^{-12} \mathrm{~m}^{2} / \mathrm{s}$ at $t=180$ days. $\alpha$ in the TFC model can be obtained based on the experimental data by the least-square fitting. $\alpha$ is 0.90 and 0.95 , when $t=90$ days and $t=180$ days in the TFC model.

In Figure 7, the numerical simulation of TFC and TC models has the similar diffusion tendency on the depth range. Although the results of these two models are close to experimental results, the simulated values of the TFC model are closer to the experimental data.

We compare the experimental and simulated chlorine concentration values to further verify the correctness of the present TFC model. A series of experiments with three mixtures (OPC only, either $30 \%$ fly ash or $70 \%$ slag as a partial replacement for Portland cement) on chloride ion penetration were carried out in [30]. We adopt the TFC model to simulate the experimental values of OPC concrete specimens at $t=3$ years and $t=8$ years. The diffusion coefficient $D=3.7178 \times 10^{-11} \mathrm{~m}^{2} / \mathrm{s}$, the surface concentration 


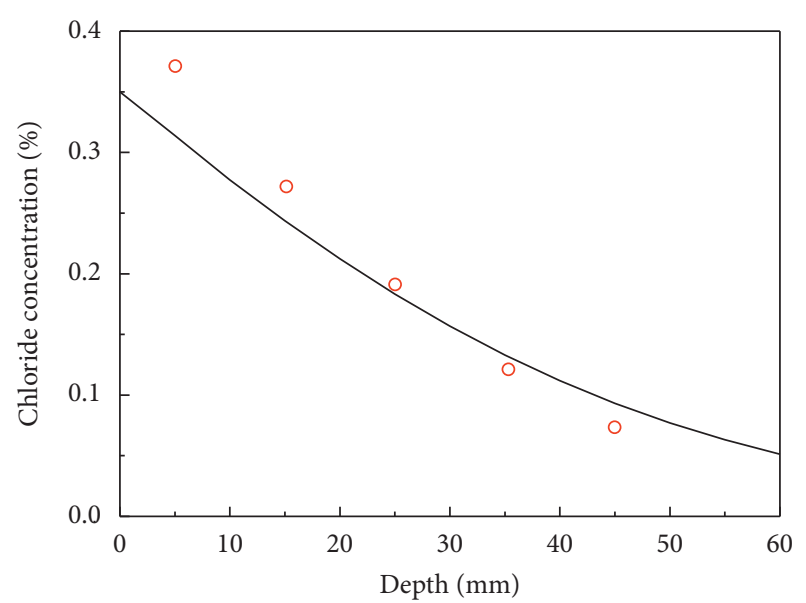

- Experimental data

- TFC

(a)

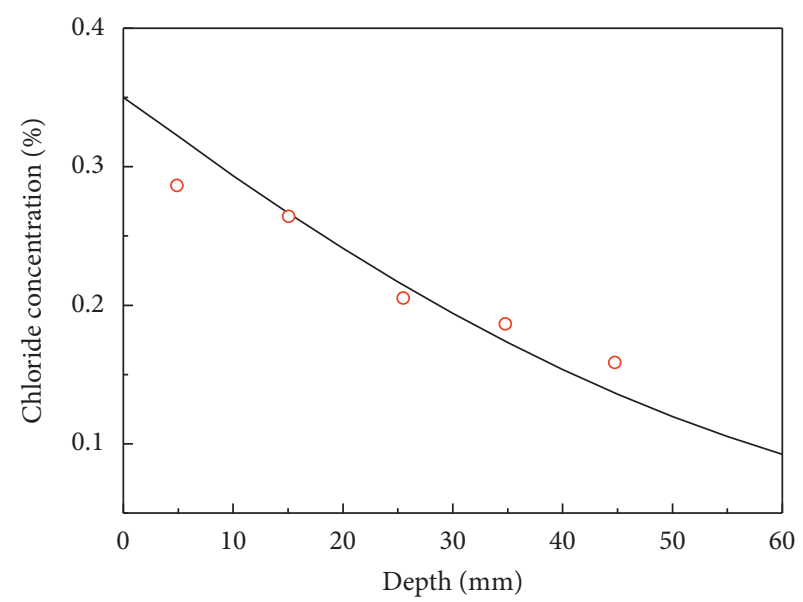

- Experimental data

TFC

(b)

Figure 8: Distribution of chloride concentration with penetration depth. (a) $t=3$ years and (b) $t=8$ years.

$C_{s}=0.35 \%$, and $\alpha=0.9$ in the TFC model. These parameter values are the same as the values in [8]. As shown in Figure 8, the numerical simulation is close to experimental results.

\section{Conclusions}

We applied the EFG meshless method to analyze the TFC diffusion model in RC. The numerical results from $1 \mathrm{D}$ and $2 \mathrm{D}$ prove that the TFC model is effective and show that the EFG meshless method is accurate and efficient. Chloride concentration drastically varies with the fractional order $\alpha$ in the TFC model. Chloride concentration at the same depth is high when $\alpha$ is high, and the initiation period of corrosion is prolonged with the increase in $\alpha$. Notably, the time-fractional diffusion equation can also be related to the fractal of the concrete microstructure, and the corresponding fractional diffusion equation can be obtained.

\section{Data Availability}

The data used to support the findings of this study are available from the corresponding author upon request.

\section{Conflicts of Interest}

The authors declare that they have no conflicts of interest.

\section{Acknowledgments}

The research described in this paper was financially supported by the Institute Foundation (ZB2018009, ZB2018007), by the Fund for Shanxi "1331 Project"Key Subjects Construction.

\section{References}

[1] P. K. Katiyar, P. K. Behera, S. Misra, and K. Mondal, "Comparative corrosion behavior of five different microstructures of rebar Steels in simulated concrete pore solution with and without chloride addition," Journal of Materials Engineering and Performance, vol. 28, no. 10, pp. 6275-6286, 2019.

[2] M. Adhvaryu, P. N. Patel, and C. D. Modhera, "Apertured EBG-based microwave patch antenna for characterization of corrosion in steel rebar of civil structures," Sensing and Imaging an International Journal, vol. 20, no. 1, pp. 20-34, 2019.

[3] M. Kurtela, V. Šimunović, I. Stojanović, and V. Alar, "Effect of the cerium (III) chloride heptahydrate on the corrosion inhibition of aluminum alloy," Materials and Corrosion, vol. 71, no. 1, pp. 1-23, 2019.

[4] S. Guzmán, J. C. Gálvez, and J. M. Sancho, "Cover cracking of reinforced concrete due to rebar corrosion induced by chloride penetration," Cement and Concrete Research, vol. 41, no. 8, pp. 893-902, 2011.

[5] A. Costa and J. Appleton, "Chloride penetration into concrete in marine environment-part II: prediction of long term chloride penetration," Materials and Structures, vol. 32, no. 5, pp. 354-359, 1999.

[6] R. Metzler and J. Klafter, "The random walk's guide to anomalous diffusion: a fractional dynamics approach," Physics Reports, vol. 339, no. 1, pp. 1-77, 2000.

[7] Tang L., Guideline for Practical Use of Methods for Testing the Resistance of Concrete to Chloride Ingress, CHLORTEST_EU Funded Research Project 'Resistance of Concrete to Chloride Ingress-from Laboratory Tests to In-Field Performance' G6RD-CT-2002-00855. Deliverable D23. Report Number: GRD1-2002-71808, 2005.

[8] S. Wei, W. Chen, and J. Zhang, "Time-fractional derivative model for chloride ions sub-diffusion in reinforced concrete," European Journal of Environment and Civil Engineering, vol. 21, no. 3, pp. 319-331, 2017.

[9] R. White, R. Robson, and K. Ness, "Anomalous anisotropic diffusion of electron swarms in AC electric fields," Australian Journal of Physics, vol. 48, no. 6, pp. 925-937, 1995.

[10] R. Gorenflo, F. Mainardi, D. Moretti, G. Pagnini, and P. Paradisi, "Discrete random walk models for space-time fractional diffusion," Chemical Physics, vol. 284, no. 1-2, pp. 521-541, 2002. 
[11] B. Li and J. Wang, "Anomalous heat conduction and anomalous diffusion in one-dimensional systems," Physical Review Letters, vol. 91, pp. 1-4, 2003.

[12] A. A. Alikhanov, "A new difference scheme for the time fractional diffusion equation," Journal of Computational Physics, vol. 280, no. 1, pp. 424-438, 2015.

[13] B. Jin, R. Lazarov, J. Pasciak, and Z. Zhou, "Error analysis of semidiscrete finite element methods for inhomogeneous timefractional diffusion," IMA Journal of Numerical Analysis, vol. 35, no. 2, pp. 561-582, 2015.

[14] M. Bitaraf and S. Mohammadi, "Analysis of chloride diffusion in concrete structures for prediction of initiation time of corrosion using a new meshless approach," Construction and Building Materials, vol. 22, no. 4, pp. 546-556, 2008.

[15] L. Guo, T. Chen, and X.-W. Gao, "Transient meshless boundary element method for prediction of chloride diffusion in concrete with time dependent nonlinear coefficients," Engineering Analysis with Boundary Elements, vol. 36, no. 2, pp. 104-111, 2012.

[16] L. Yao, L. Zhang, L. Zhang, and X. Li, "Prediction of initiation time of corrosion in RC using meshless methods," Computers and Concrete, vol. 16, no. 5, pp. 669-682, 2015.

[17] L. Yao, X. Li, L. Zhang, and L. Zhang, "Prediction of chloride diffusion in concrete structure using meshless methods," Advances in Materials Science and Engineering, vol. 2016, Article ID 3824835, 10 pages, 2016.

[18] A. Graça, R. P. R. Cardoso, and J. W. Yoon, "Subspace analysis to alleviate the volumetric locking in the 3D solid-shell EFG method," Journal of Computational and Applied Mathematics, vol. 246, pp. 185-194, 2013.

[19] J. Belinha, A. L. Araújo, A. J. M. Ferreira, L. M. J. S. Dinis, and R. M. Natal Jorge, "The analysis of laminated plates using distinct advanced discretization meshless techniques," Composite Structures, vol. 143, pp. 165-179, 2016.

[20] H. Salari-Rad, M. Rahimi-Dizadji, S. Rahimi-Pour, and M. Delforouzi, "Meshless EFG simulation of linear elastic fracture propagation under various loadings," Arabian Journal for Science and Engineering, vol. 36, no. 7, pp. 13811392, 2011.

[21] S. Nakagawa and V. A. Korneev, "Effect of fracture compliance on wave propagation within a fluid-filled fracture," The Journal of the Acoustical Society of America, vol. 135, no. 6, pp. 3186-3197, 2014

[22] S. Rahman and B. N. Rao, "Probabilistic fracture mechanics by Galerkin meshless methods? part II: reliability analysis," Computational Mechanics, vol. 28, no. 5, pp. 365-374, 2002.

[23] Y. P. Chen, A. Eskandarian, M. Oskard, and J. D. Lee, "Meshless simulation of crack propagation in multiphase materials," Theoretical and Applied Fracture Mechanics, vol. 45, no. 1, pp. 13-17, 2006.

[24] J. N. Vyas and S. Daxini, "Meshless formulation and parameters study for an elastic bar problem," in Proceedings of the 2016 International Conference on Electrical, Electronics, and Optimization Techniques (ICEEOT), Chennai, India, March 2016.

[25] T. Belytschko, Y. Y. Lu, and L. Gu, "Element-free Galerkin methods," International Journal for Numerical Methods in Engineering, vol. 37, no. 2, pp. 229-256, 1994.

[26] Y. Y. Lu, T. Belytschko, and L. Gu, "A new implementation of the element free Galerkin method," Computer Methods in Applied Mechanics \& Engineering, vol. 113, no. 3-4, pp. 397-414, 1994.

[27] H. Sun, W. Chen, H. Sheng, and Y. Chen, "On mean square displacement behaviors of anomalous diffusions with variable and random orders," Physics Letters A, vol. 374, no. 7, pp. 906-910, 2010.

[28] L. Tang and J. Gulikers, "On the mathematics of time-dependent apparent chloride diffusion coefficient in concrete," Cement \& Concrete Research, vol. 37, no. 4, pp. 589-595, 2007.

[29] Z. Szweda, "Comparison of protective properties of concretes with low alkali cement," IOP Conference Series: Materials Science and Engineering, vol. 603, Article ID 042059, 2019.

[30] M. D. A. Thomas and P. B. Bamforth, "Modelling chloride diffusion in concrete," Cement and Concrete Research, vol. 29, no. 4, pp. 487-495, 1999. 